\title{
Multiple Beam Electron Gun Development for High-Power Amplifiers
}

\author{
K. T. Nguyen (KN Research, Silver Spring, MD 20905. Email: nguyen@estd.nrl.navy.mil ) \\ D. E. Pershing (MRC), J. Pasour (MRC) and J. Petillo (SAIC)
}

A very attractive class of amplifiers that has the potential to provide high RF power at low beam voltage uses multiple electron beams rather than the single beam of conventional devices. These multiple beam amplifiers (MBAs) typically have individual beam channels with a common RF interaction region. Figure 1, which shows a multiple beam klystron (MBK), illustrates the concept. In such a scheme, individual beamlets have parameters similar to those in conventional, single-beam amplifiers, while multiplying the output power by approximately the number of beamlets. The important result is that the operating voltage and space charge limitations for each beamlet are essentially unchanged, yet the output power can be much greater than that of a corresponding single-beam device. Furthermore, the overall high perveance characteristic of the MBA permits large bandwidth operation, while the low perveance of the individual beamlet enables high efficiency operation.

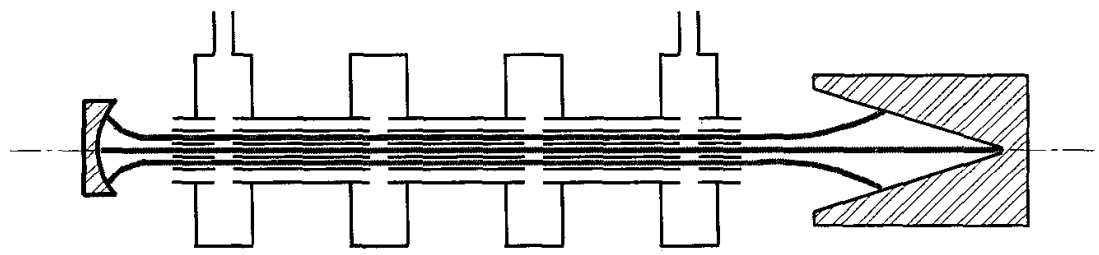

Figure 1: Schematic Diagram of a Multiple Beam Klystron.

These advantages are a key reason for the extensive MBA research and development effort in Russia for a variety of applications, including radar and communications. Of particular interest is the work at Istok where many thousands of MBKs have been fabricated [1]. In recent years, MBA developments have also been initiated in China and France $[2,3]$. Of course, the advantages mentioned above come at the cost of added complexity, especially concerning beam optics (generation and transport). Thus, beam optics is the main objective of the present work, which focuses on the design of an electron gun for an S-band amplifier. The design goals include a total beam current of 35-40 A with a beam voltage of $40-45 \mathrm{kV}$ and a cathode loading below $10 \mathrm{~A} / \mathrm{cm}^{2}$ (for lifetime considerations). A preliminary gun design is shown in Figure 2, illustrating electron trajectories calculated using MICHELLE [4] for an eight-beam electron gun topology. Also visible in the figure is the outline of the mesh employed in the simulation. This mesh has been generated using the ICEM-CFD [5] mesh generator. The eight-beam gun topology permits the simulation of just a $90^{\circ}$ wedge of the gun by taking advantage of the periodic boundary condition available in MICHELLE as shown in the figure. This particular design meets the target goal of a $36 \mathrm{~A}$ total beam current at a beam voltage of $45 \mathrm{kV}$, and is achieved with a control electrode voltage of $1.3 \mathrm{kV}$. It should be noted that in multiple beam guns the control electrode plays a key role in the simultaneous control of both the beam current (via space-chargelimited emission) and beam convergence (laminarity).

Figure 3 shows a full eight-beam simulation with a much longer beam tunnel. The figure shows that the beamlets are first focused electrostatically to a waist then expand due to space-charge forces in the absence of restoring forces from applied magnetic fields, as is expected. For the present design, Brillouin focusing is employed, which involves shielding the cathode-anode region and abruptly turning on the magnetic field at the beam waist. To ensure good beam transport, it is critical that the local magnetic fields be axisymmetric around the minor axis of each beamlet. This is achieved in the present design as illustrated in Figure 4, which shows beamlet trajectories with a magnetic field that was 
calculated using MAXWELL. In this Figure, the peak-applied magnetic field is about $1.1 \mathrm{kG}$, corresponding to about 1.4 of the Brillouin value.

Detailed results and design approach will be presented at the conference.

*This research is supported in part by an SBIR from the Office of Naval Research.

\section{References:}

1. E.A. Gelvich, "Multiple Beam Amplifiers: A Review," presented at MBA workshop, SAIC, McLean, VA, May 18,2001 .

2. D. Yaogen, P. Jun, Z. Yunshu, and S. Shaominh, "Theoretical and Experimental research on Multi-beam

Klystron," High Energy Density Microwavesî, AIP Conf. Proc. 474, p.127, (Oct. 1998).

3. C. Bearzatto, A. Beunas, and G. Faillon, " Long Pulse and Large Bandwidth Multi-beam Klystron," High Energy Density Microwaves, AIP Conf. Proc. 474, p.107, (Oct. 1998).

4. J.Petillo, et al., i The new 3D Electron Gun and Collector Modeling Tool: MICHELLE, î Proc. of the $2^{\text {nd }}$ IEEE IVEC (Noorwijk, Netherlands), p.199, (April 2001).

5. ICEM-CFD Engineering, 2855 Telegraph Ave., Berkeley, CA 94705

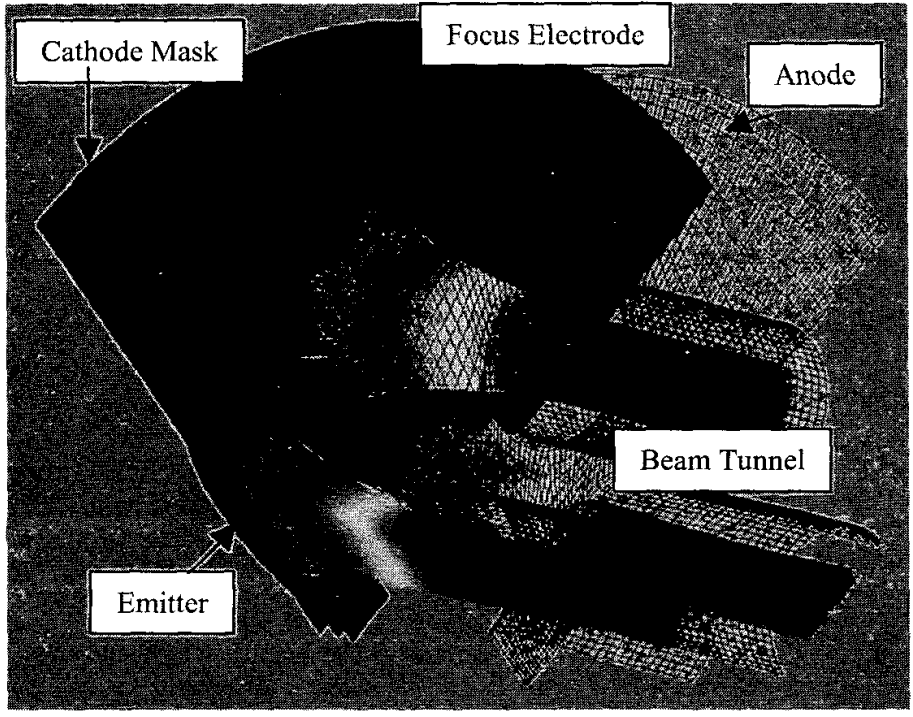

Figure 2: Cut away view of an eight-beam gun

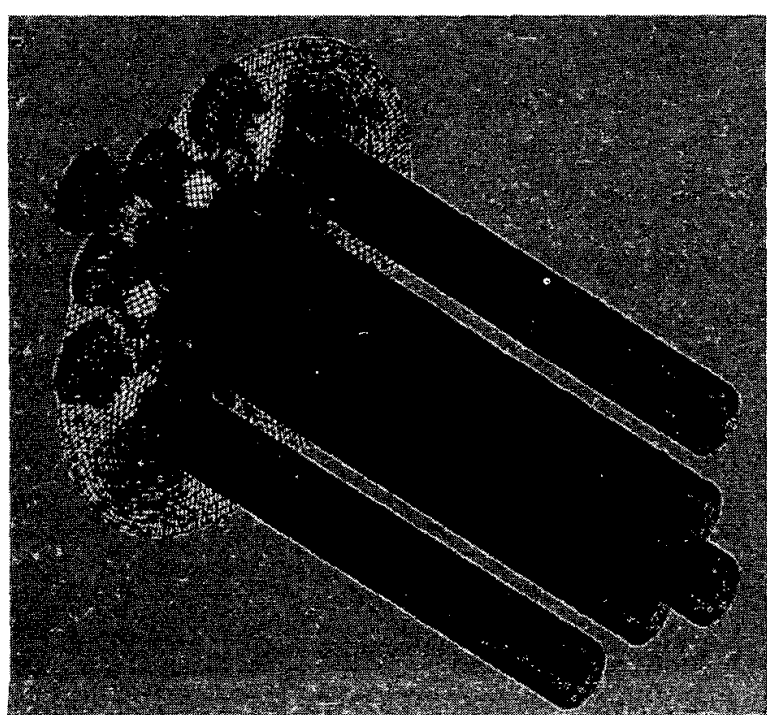

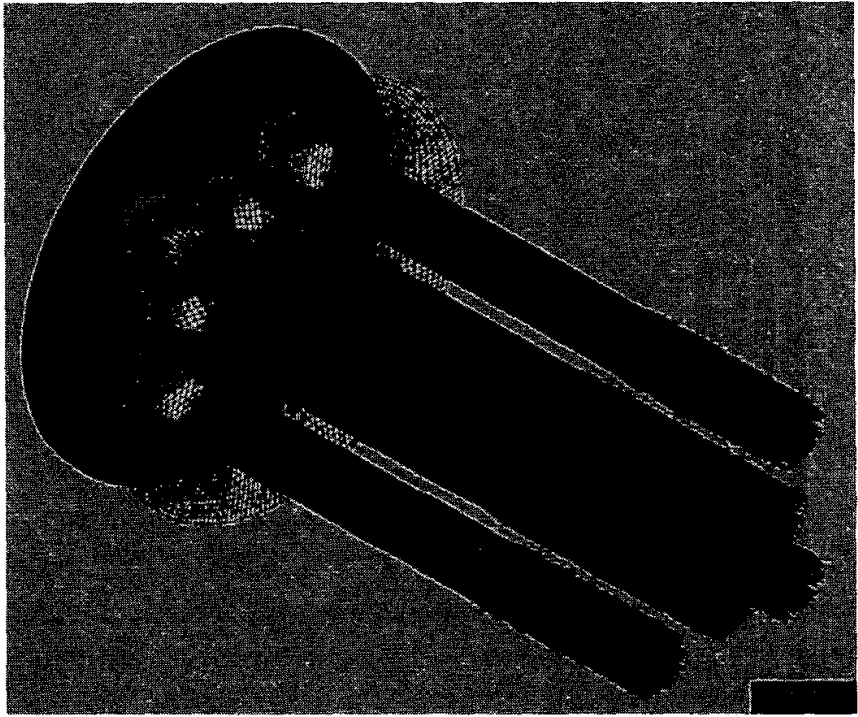

Figure 3: Full view of the eight-beam preliminary design from a MICHELLE gun simulation with no magnetic fields.

Figure 4: Isometric view of beamlets with magnetic field. The magnetic system was design using MAXWELL. The beam tunel length in this simulation is 1.2 cyclotron period. 